\title{
Social capital and suicide: an ecological study in Tokyo, Japan
}

\author{
Masumi Okamoto $\cdot$ Norito Kawakami • \\ Yoshifumi Kido $\cdot$ Keiko Sakurai
}

Received: 10 January 2012/ Accepted: 8 November 2012/Published online: 23 November 2012

(C) The Japanese Society for Hygiene 2012

\begin{abstract}
Objective The aim of this study is to clarify the association between area-based social capital and suicide rate among municipalities of Tokyo.

Methods The study areas were 20 administrative municipalities of Tokyo. Social capital (i.e., organizational membership, social trust, fairness, helpfulness, and confidence in organizations) was measured based on data from a previous survey (response rate $28 \%$ ). Gender-specific ageadjusted suicide rates averaged over 5 years, sociodemographic, and other area characteristics were obtained from relevant national statistics. Multiple linear regression analysis of suicide rates was applied on each social capital variable, adjusting for the other area characteristics.

Results There was no significant crude Pearson's correlation between any social capital variable and suicide rate. Multiple regression analyses revealed a significant negative association between social trust and suicide rate for men $(p=0.04)$.

Conclusions While based on only limited evidence from a cross-sectional ecological study, area-based social trust
\end{abstract}

M. Okamoto $(\square) \cdot$ N. Kawakami

Department of Mental Health, School of Public Health, The University of Tokyo, 7-3-1 Hongo, Bunkyo-ku,

Tokyo 113-0033, Japan

e-mail: okamoto510@gmail.com

\section{Y. Kido}

Department of Psychiatric Nursing, Graduate School of Medicine, The University of Tokyo, 7-3-1 Hongo, Bunkyo-ku, Tokyo 113-0033, Japan

\section{K. Sakurai}

Department of Health Economics and Epidemiology Research, School of Public Health, The University of Tokyo, 7-3-1 Hongo, Bunkyo-ku, Tokyo 113-0033, Japan may be associated with decreased suicide rates for men in Tokyo.

Keywords Suicide $\cdot$ Mental health - Social capital · Social trust $\cdot$ Community

\section{Introduction}

Social capital is defined as participation, trust, networks, and cooperation, enabling people in communities to take positive social action [1-3]. Many researchers have taken social capital into consideration in attempts to identify factors that improve the health status of people in communities and implement proper intervention to deal with various health issues. Additionally, researchers have identified the association between social capital and better mental health as well as other health outcomes in the community [4-7]. Several systematic reviews have indicated that social capital, assessed at the individual level, is consistently associated with better mental health, including psychiatric symptoms and mental disorders [5]. Among these studies, cognitive social capital, such as trust in neighbors, was associated with mental health more so than structural social capital, such as participation in community activities [5, 8, 9]. A fewer studies have shown that area-level social capital was associated with mental health $[5,7,9]$.

However, a more recent study found that area-level social capital is associated with common mental disorders in deprived communities of the UK [10,11]. Additionally, cognitive social capital (trust) and structural social capital, both assessed at the area level, have been found to be significantly associated with mental health as measured by the Short Form-36 (SF-36) in a national sample of community residents in Japan [12]; the effect was greater than 
with individual-level social capital variables. Researchers also propose that social capital might be useful in preventing suicide [13-15]. However, few studies have investigated the link between social capital and suicide. Kelly et al. [16] investigated the association between social capital and suicide rate in 11 European countries and found that social trust was associated with lower suicide rate at country level, while no clear association was seen for other social capital indicators, such as fairness, helpfulness, participation in activities, and trust in organizations and regulations. Another interesting study of suicide rate among psychiatric patients, discharged from a hospital, reported that state-level social capital was associated with lower suicide rate in the USA [17]. On the other hand, one unpublished study, introduced by a systematic review [5], unexpectedly found that the number of membership organizations per capita aggregated at area level was associated with greater suicide rates in metropolitan areas. Thus, it is still unclear whether social capital in the community is associated with lower suicide risk. In addition, previous studies that yielded positive findings $[16,17]$ used a relatively large area, such as country or state, as the unit of analysis; a larger area is often heterogeneous and may lead to no clear finding. Further, using a larger area might be vulnerable to potential confounding factors, such as a national/regional policy or the mental health system. Therefore, findings should be replicated using a smaller area as the unit, as well as more diverse social and cultural settings.

The aim of this study is to clarify the inverse association between area-based social capital and suicide rates in the community among selected cities/municipalities in Tokyo, Japan via a cross-sectional, ecological study design.

\section{Methods}

\section{Study population}

We used data from a previous survey conducted in 2009, which primarily intended to examine the association between social capital and stigma toward people with mental illness in Tokyo, Japan. The researchers selected 20 administrative areas of Tokyo, including 12 of the 23 wards (i.e., Bunkyo, Itabashi, Nerima, Adachi, Taito, Sumida, Koutou, Shibuya, Shinagawa, Setagaya, Suginami, and Nakano), 6 of 26 suburb cities (Akiruno, Musashino, Chofu, Hino, Mizuho, and Hinohara), and 2 of 4 islands (Hachizyo and Ohshima) as target areas. Inclusion criteria initially included having a rehabilitation workshop(s) for people with mental illness in a particular area, since the survey initially intended to associate community social capital and self-stigma among people with mental disorders in these workshops, and the availability of the voter registration list or resident register list for a survey. A random sample of 100 residents, aged 20 years and above, was selected in each area from the voter registration list. A questionnaire was sent to the total 2,000 subjects by mail. The study aims and procedure were informed by letter, asking those who agreed to participate in the study to complete and return the questionnaire. The study aims and procedures were reviewed and approved by the Ethical Committee of Faculty of Medicine, The University of Tokyo, Japan. A mailed questionnaire, including social capital variables, was sent to participants, and a total of 554 responses were returned (response rate $28.2 \%$ ) after excluding those who did not complete the information on gender, age, and social capital variables. The number of respondents by area was 27.7 on average and ranged from 20 to 39 ; the response rate ranged from 20 to $40 \%$. The low response rate was partly attributable to the sensitive topic of the survey, i.e., stigma toward people with mental disorder, which may have made people less interested or even reluctant to complete the questionnaire.

\section{Measures}

\section{Measurement of social capital}

We used five questions on structural and cognitive social capital that had been used in a previous study [18]. These questions were originally in the US General Social Survey conducted by the National Opinions Research Center (Chicago, IL, USA) and the World Values Survey conducted by the World Values Survey Association (Stockholm, Sweden), and have been used frequently in previous studies on social capital [19]. These surveys were translated into several Asian languages including Japanese to be used in the Asia \& Pacific Values Survey that was conducted by The Institute of Statistical Mathematics (Tokyo, Japan) [18].

\section{Organizational membership}

Organizational membership, a dimension of structural social capital, was measured by a 1-item question [18]. The question asked about participation in one or more voluntary organizations including sports, hobbies, literature groups, alumni associations, religious groups, political organizations, etc. The response to this item was converted into a dichotomous variable ( $1=$ belonging to any, $0=$ none $)$. The proportion of those who belonged to any type of organization was calculated and used as the area-based indicator of organizational membership. 


\section{Cognitive social capital}

Cognitive social capital was measured via four items [18]. A single question on social (or interpersonal) trust asked, "Generally speaking, would you say that most people can be trusted or that you can't be too careful in dealing with people?" with four possible response options: "Can be trusted," "Can't be too careful," "Other," and "Don't know." Responses were dichotomized $(1=$ Can be trusted; $0=$ Can't be too careful, Other, and Don't know). Another question concerning perceived fairness asked, "Do you think that most people would try to take advantage of you if they had the chance or would they try to be fair?" with 4 possible response options: "Take advantage," "Try to be fair," "Other," and "Don't know." Responses were dichotomized $(1=$ Try to be fair; $0=$ Take advantage, Other, and Don't know). A question concerning perceived helpfulness of people asked "Generally speaking, would you say that most of the time, people try to be helpful or are they mostly just looking out for themselves?" with 4 possible response options: "Try to be helpful," "Look out for themselves," "Other," and "Don't know." Responses were dichotomized $(1=$ Try to be helpful; $0=$ Look out for themselves, Other, and Don't know). The average number of each question, by area, was calculated and used as the area-based indicator for cognitive social capital.

\section{Confidence in organizations}

The last question concerned confidence in organizations, a dimension of cognitive social capital, and asked whether respondents felt confidence in each of 10 institutions/ organizations ("religious," "the law and legal system," "press and television," "police," "national assembly," "nonprofit and nongovernmental organizations," "social welfare facilities," "United Nations," and "science and technology") with five response options: "Very confident," "Somewhat confident," "Not confident," "Not confident at all," and "Don't know." According to previous studies, the number of positive responses (i.e., "Very confident" or "Somewhat confident") was counted. The score ranged from 0 to 10, with higher score being indicative of higher confidence in institutions. The average score, by area, was used as an area-based indicator of confidence in organizations.

\section{Suicide rate}

Suicide rate could be unstable if the area population is small, and it is sometimes difficult to calculate age-adjusted suicide rates in such an area. Thus, we calculated the 5 -year average of age-adjusted suicide rates for each area from 2003 to 2007 based on National Census data, separately for men and women.

Sociodemographic variables

We also used data on the following area-based sociodemographic characteristics, which might be associated with suicide rate [20]: total population size [21] and population density (persons/square $\mathrm{km}$ ) [22], the proportion of elderly, aged 65 years or over (\%) [20], proportion of workers engaged in primary industry (i.e., agriculture and fisheries) (\%) [23], unemployment rate (\%) [24], and number of psychiatrists (number per 100,000 population). These data were only available for all target areas for 2005 or 2006 and were based on the National Census 2005, the National Survey of Medical Institutions and Doctors, Dentists, Pharmacists Survey 2006, and other relevant information sources.

\section{Statistical methods}

Pearson's correlation coefficients were calculated between the five area-based social capital variables, sociodemographic characteristics of areas, and age-adjusted suicide rates, separately for men and women $(N=20)$. Multiple linear regression analysis was conducted for age-adjusted suicide rates on each of the area-based social capital variables, adjusting for population, population density, unemployment rate, proportion of elderly, proportion of primary industry workers, and number of psychiatric doctors, separately for men and women. The analyses were conducted using PROC CORR and PROC REG, SAS version 9.1.3 for Windows (SAS Inc., Cary, NC). Significance level was set at less than 0.05 for all tests.

\section{Results}

Table 1 presents the sociodemographic characteristics and social capital variables of the 20 areas included in this study. There was no significant correlation between any area-based social capital variable and age-adjusted suicide rate for men or women (Table 2). Response rate by area was not significantly associated with age-adjusted suicide rate for men or women. In the multiple regression analysis, age-adjusted suicide rate was significantly and inversely associated with social trust for men (Table 3; $\beta=-0.92, p=0.04$ ). Additionally, other social class variables were not significantly associated for men (data available upon request). For women, no significant association was observed between any social capital variable and suicide rate (data available upon request). These patterns were the same when the two islands were excluded from the analysis (data available upon request). 


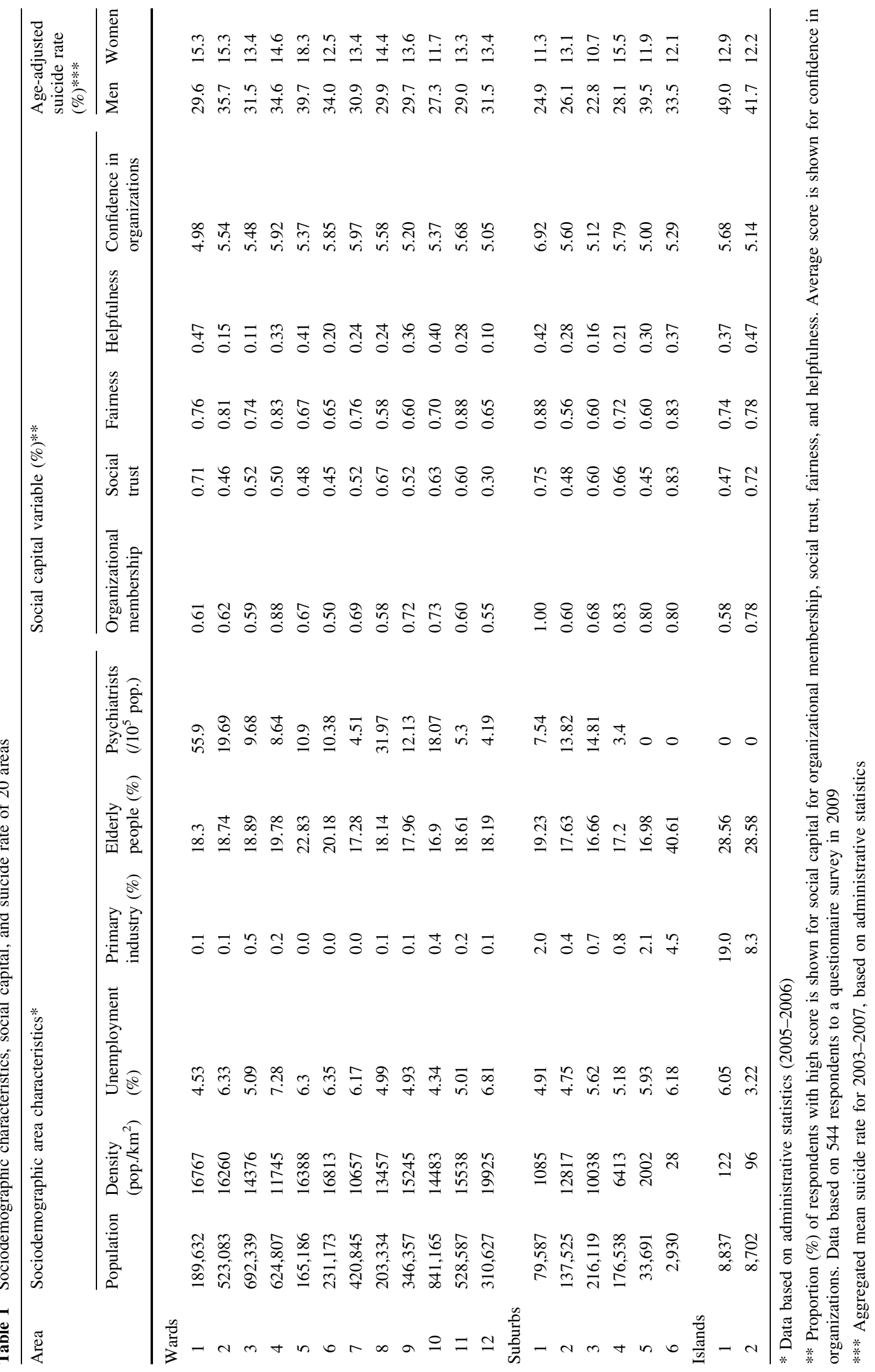


Table 2 Pearson's coefficients $(r)$ between area-based social capital variables and sociodemographic characteristics and age-adjusted suicide rate in 2003-2007 for men and women in 20 cities/municipalities of Tokyo, Japan: an ecological analysis

\begin{tabular}{|c|c|c|c|c|}
\hline & \multicolumn{2}{|c|}{$\begin{array}{l}\text { Men } \\
(n=20)^{*}\end{array}$} & \multicolumn{2}{|c|}{$\begin{array}{l}\text { Women } \\
(n=20)^{*}\end{array}$} \\
\hline & $r$ & $p$ & $r$ & $p$ \\
\hline \multicolumn{5}{|c|}{ Sociodemographic characteristics in $2005 / 2006$} \\
\hline Population & -0.308 & 0.19 & 0.091 & 0.70 \\
\hline Population density & -0.348 & 0.13 & 0.449 & 0.05 \\
\hline Unemployment & 0.226 & 0.34 & 0.241 & 0.31 \\
\hline Primary industry & 0.708 & 0.00 & -0.231 & 0.33 \\
\hline Elderly people & 0.496 & 0.03 & -0.107 & 0.65 \\
\hline Psychiatrists ( $/ 10^{5}$ pop.) & -0.337 & 0.15 & 0.313 & 0.18 \\
\hline \multicolumn{5}{|l|}{ Social capital in 2009} \\
\hline Organizational membership (\%) & -0.130 & 0.58 & -0.204 & 0.39 \\
\hline Social trust $(\%)$ & -0.266 & 0.26 & -0.212 & 0.37 \\
\hline Fairness $(\%)$ & 0.099 & 0.68 & 0.015 & 0.95 \\
\hline Helpfulness (\%) & 0.256 & 0.28 & 0.027 & 0.91 \\
\hline $\begin{array}{l}\text { Confidence in } \\
\text { organizations (score) }\end{array}$ & -0.192 & 0.42 & -0.080 & 0.74 \\
\hline
\end{tabular}

$* N=20$ indicates the number of cities or municipalities of Tokyo, Japan

Table 3 Multiple linear regression of age-adjusted suicide rate in 2003-2007 on social trust and other sociodemographic characteristics for men and women in 20 cities/municipalities of Tokyo, Japan: An ecological analysis

\begin{tabular}{|c|c|c|c|c|}
\hline & \multicolumn{2}{|c|}{$\begin{array}{l}\text { Men } \\
(n=20)^{* *}\end{array}$} & \multicolumn{2}{|c|}{$\begin{array}{l}\text { Women } \\
(n=20)^{* *}\end{array}$} \\
\hline & $\beta$ & $p$ & $\beta$ & $p$ \\
\hline Social trust $(\%)^{*}$ & -0.920 & 0.039 & -0.023 & 0.971 \\
\hline Population & 0.139 & 0.533 & -0.175 & 0.617 \\
\hline Population density (pop. $/ \mathrm{km}^{2}$ ) & -0.524 & 0.255 & 0.461 & 0.518 \\
\hline Unemployment rate $(\%)$ & -0.134 & 0.567 & 0.213 & 0.563 \\
\hline Primary industry workers (\%) & 0.258 & 0.414 & 0.021 & 0.966 \\
\hline Proportion of elderly (\%) & 0.634 & 0.051 & 0.152 & 0.749 \\
\hline Psychiatrists (/10 5 pop.) & 0.376 & 0.203 & 0.270 & 0.551 \\
\hline
\end{tabular}

* Adjusted for population, population density, unemployment rate, ratio of primary industry, ratio of elderly people, and number of psychiatrists

** $N=20$ indicates the number of cities or municipalities of Tokyo, Japan

\section{Discussion}

We found a significant inverse association between areabased social trust, an indicator of cognitive social capital, and age-adjusted suicide rate for men among 20 cities/ municipalities of Tokyo, Japan, after adjusting for major sociodemographic characteristics of the areas. The other social capital indicators were not significantly associated with suicide rates of men or women. This finding is similar to that of a previous study among 11 European countries that found a significant inverse association between country-level social trust and suicide rate [16]. The present study confirms the previous observation and expands it at least for men in Tokyo, Japan, using cities/municipalities as the unit of analysis, where the variety of healthcare policies and systems should be much smaller than across countries. The present findings are also consistent with previous research finding that state-level social capital, as measured by a composite index, was associated with lower suicide rate among psychiatric patients who were discharged from a hospital in the USA [17]. While these is still limited evidence, these findings, together with the current findings, suggest that area-level social capital, particularly social trust, is associated with lower suicide rates. However, since the significant association between area-based social trust and suicide rate was only observed after adjusting for sociodemographic characteristics, the association may be modest, if any.

Previous studies on social capital assessed at the individual level and mental health have indicated that cognitive social capital, including social trust, is associated with mental health more so than structural social capital [5]. The present finding, as well as a past finding [16], which suggest social trust was the most associated with suicide, is in line with these observations. Social trust is expected to play a central role in developing emotional support and mutual respect in the community [7]. These are important factors to reducing or buffering emotional reactions, such as anxiety and depression. This may be a reason why social trust was most associated with lower suicide rates. On the other hand, one unpublished study [5] found that the number of membership organizations, per capita aggregated to area level, was associated with greater suicide rates in metropolitan areas. However, we observed a nonsignificant but inverse association between organization membership and suicide rate for men and women. A recent study in Japan also showed that structural social and cognitive capital, at the area level, was significantly associated with mental health [12]. Further investigation is needed to examine the association of structural social capital and suicide.

In the present study, social trust was associated with low suicide rates for men, but not for women; however, a nonsignificant inverse association between the two variables was observed for both men and women in the crude analysis. Why was social trust associated with suicide rates for men more so than for women? There may be two reasons. First, the impact of social capital on suicidal behaviors (such as ideation and attempt) may differ by gender. A previous study found that area-based social 
capital was more protective for suicidal ideation and attempt among female than male students [25]. The authors argued that social capital may be more important for preventing suicide among women than men, as it is so for health in general [26]. Second, previous ecological studies in Japan revealed that suicide was influenced by economic status among men [24]. According to a lifestyle survey in Tokyo, average household income has decreased since 1998 (data on statistics for Tokyo). Further, a traditional gender role in Japan requires men to be financially responsible in supporting their family $[27,28]$. The feeling of not fulfilling this most important duty for men may decrease self-esteem and confidence among men in Japan, thus causing greater psychological distress. Under such a critical situation for men, related to the current economic recession, better community social trust might be more effective in reducing distress and preventing suicide. This notion is also supported by a previous finding that arealevel social capital is associated with common mental disorders only in deprived communities [11]. Further, social trust may be associated more so with a reduced risk of suicide for men in Japan who have faced economic difficulty. This hypothesis on the interaction between socioeconomic adversities and social capital, in terms of mental health and suicide, should be examined further.

The strength of this study is that it is the first to examine the association between social capital and suicide in Japan using a smaller unit (i.e., cities/municipalities) [16, 17]. However, there are several limitations of this study that should be noted. First, the study areas did not represent all cities/municipalities of Tokyo, rather being selected by the presence of rehabilitation workshops. As such, areas under study might be biased to a community with more welfareoriented policies and budgets, which might result in less clear associations. Second, the timing of data collection of social capital, sociodemographic, and suicide rate did not match completely. Data on suicide and sociodemographic characteristics were collected based on statistics from $2006 / 2007$, before the survey on social capital in 2009 , because of the limited availability of data, particularly for smaller municipalities. For these reasons, the association may be underestimated because of time difference in the measurement of exposure and outcome, and causality could be reverse, if any. Third, since data on income and education attainment were not available for every area, we could not adjust for these socioeconomic status (SES) variables in the analysis; therefore, we adjusted for only unemployment rate. These SES variables might confound the association between social capital and suicide. Forth, the response rate of the survey on social capital was low (28\%). Although area response rates were not associated with suicide rates in this study, greater participation of those who had more interest in the survey might lead to over- or underestimation of the association. Fifth, this was a community-level ecological study, adjusting for the limited sociodemographic variables. Therefore, we could not eliminate the possibility of some area characteristics confounding the association. Finally, this study was conducted among cities/municipalities in Tokyo. Especially in urban life, people tend to move around for their work and social activities across borders of administrative areas, much more so than in rural areas. The impact of area-based social capital on suicide may have become weaker in this study. This should be investigated further using rural and urban areas and including indicators of people's mobility in the analysis, or the study should be conducted longitudinally, comparing baseline data with data collected in later years.

We conclude that social capital, particularly social trust, might be associated with low suicide rates for men in cities/ municipalities of Tokyo, Japan. Research should be conducted in order to replicate the present finding and adjust for income and educational attainment as well as other covariates, to use data collected in the same year, and to examine the association between social capital components and gender- and age-specific suicide rates. Research is also needed to explore a possible mechanism in the link between social capital and suicide rates, with a particular focus on a buffering effect.

Acknowledgments This study was partly funded by a Grant-in-Aid for Scientific Research on Innovative Areas (Research in a Proposed Research Area) 2009-2011 (no. 4102-21119001) from the Ministry of Education, Culture, Sports, Science, and Technology, Japan.

\section{References}

1. Putnam RD. Making democracy work. Civic traditions in modern Italy. Princeton: Princeton University Press; 1993.

2. Baum F. Social capital: is it good for your health? Issues for public health agenda. J Epidemiol Community Health. 1999;53: 195-6.

3. Lochner K, Kawachi I, Kennedy BP. Social capital: a guide to its measurement. Health Place. 1999;5:259-70.

4. Almedom AM. Social capital and mental health: an interdisciplinary review of primary evidence. Soc Sci Med. 2005;61: 943-64.

5. De Silva MJ, McKenzie K, Harpham T, Huttly SR. Social capital and mental illness: systematic review. J Epidemiol Community Health. 2005;59:619-27.

6. Whitney R, McKenzie K. Social capital and psychiatry: review of the literature. Harv Rev Psychiatr. 2005;13:71-84.

7. Kim D. Blues from the neighborhood? Neighborhood characteristics and depression. Epidemiol Rev. 2008;30:101-17.

8. Phongsavan P, Chey T, Bauman A, Brooks R, Silove D. Social capital, socio-economic status and psychological distress among Australian adults. Soc Sci Med. 2006;63(10):2546-61.

9. Yip W, Subramanian SV, Mitchell AD, Lee DT, Wang J, Kawachi I. Does social capital enhance health and well-being? Evidence from rural China. Soc Sci Med. 2007;64(1):35-49. 
10. McCulloch A. Social environments and health: a cross sectional survey. Br Med J. 2001;323:208-9.

11. Stafford M, De Silva M, Stansfeld S, Marmot M. Neighbourhood social capital and common mental disorder: testing the link in a general population sample. Health Place. 2008;14(3):394-405.

12. Hamano T, Fujisawa Y, Ishida Y, Subramanian SV, Kawachi I, Shiwaku K. Social capital and mental health in Japan: a multilevel analysis. PLoS ONE. 2010;5(10):e13214.

13. Mignone J, O’Neil J. Social capital and youth suicide risk factors in first nations communities. Can J Public Health. 2005;96:S51-4.

14. Van Hooijdonk C, Droomers M, Deerenberg IM, Mackenbach JP, Kunst AE. The diversity in associations between community social capital and health per health outcome, population group and location studied. Int J Epidemiol. 2008;37:1384-92.

15. Patel V. Building social capital and improving mental health care to prevent suicide. Int J Epidemiol. 2010;39:1411-2.

16. Kelly BD, Davoren M, Mhaolain AN, Breen EG, Casey P. Social capital and suicide in 11 European countries: an ecological analysis. Soc Psychiatry Psychiatr Epidemiol. 2009;44:971-7.

17. Desai RA, Dausey DJ, Rosenheck RA. Mental health service delivery and suicide risk: the role of individual patient and facility factors. Am J Psychiatry. 2005;162:311-8.

18. Yamaoka K. Social capital and health and well-being in East Asia: a population-based study. Soc Sci Med. 2008;66:885-99.

19. Kawachi I, Kennedy BP, Lochner K, Prothrow-Stith D. Social capital and income inequality, and mortality. Am J Public Health. 1997;87:1491-8.
20. Aihara H, Iki M. An ecological study of the relations between the recent high suicide rates and economic and demographic factors in Japan. J Epidemiol. 2002;13(1):56-61.

21. McCall PL, Tittle CR. Population size and suicide in US cities: a static and dynamic exploration. Suicide Life Threat Behav. 2007;37(5):553-64.

22. Stark C, Hopkins P, Gibbs D, Belbin A, Hay A. Population density and suicide in Scotland. Rural Remote Health. 2007; 7(3):672.

23. Stafford M, Cummins S, Macintyre S, Ellaway A, Marmot M. Gender differences in the associations between health and neighbourhood environment. Soc Sci Med. 2005;60:1681-92.

24. Nishimura M, Terao T, Soeda S, Nakamura J, Iwata N, Sakamoto K. Suicide and occupation: further supportive evidence for their relevance. Prog Neuro- Psychopharmacol Biol Psychiatry. 2004;28: 83-7.

25. Langille DB, Asbridge M, Kisely S, Rasic D. Suicidal behaviours in adolescents in Nova Scotia, Canada: protective associations with measures of social capital. Soc Psychiatry Psychiatr Epidemiol. 2011. doi:10.1007/s00127-011-0461-x.

26. Takei N, Kawai M, Mori N. Sluggish economics affect health of Japanese business warriors. Br J Psychiatry. 2000;176:494-5.

27. Harris PB, Long SO, Fujii M. Men and elder case in Japan: a ripple of change? J Cross-Cult Gerontol. 1998;3:177-98.

28. Kitamura Y. Gender equality dilemma in Japanese society: how traditional ideas affect both women and men. Bunkyodaigaku kokusaigakubu kiyou. 2008;19(1):65-78. 